Int. J. Morphol.,

37(4):1517-1521, 2019.

\title{
Análisis del Lexema Neur(o) y sus Derivados en el Aprendizaje de la Anatomía
}

\author{
Analysis of Neur(o) Lexeme and its Derivatives in Anatomy Learning
}

\author{
Nicolás Vidal-Seguel ${ }^{1,2,3}$; Marcela Pumeyrau Solar ${ }^{4}$; Rodrigo Lizama Pérez ${ }^{3,5}$; \\ Rodrigo Muñoz Cofré ${ }^{6}$; Daniel Conei ${ }^{6,7}$; Christopher Nicholson ${ }^{8}$ \& Mariano del Sol $^{6}$
}

\begin{abstract}
VIDAL-SEGUEL, N.; PUMEYRAU, S. M.; LIZAMA, P. R.; MUÑOZ, C. R.; CONEI, D.; NICHOLSON, C. \& DEL SOL, M. Análisis del lexema neur(o) y sus derivados en el aprendizaje de la anatomía. Int. J. Morphol., 37(4):1517-1521, 2019.

RESUMEN: La neuroanatomía es una de las ramas más complejas de abordar por los estudiantes por su lenguaje complejo y extenso. Es por ello que el término neur(o) podría favorecer su aprendizaje, debido a que múltiples conceptos y términos lo tienen incorporado como prefijo, sufijo o interfijo. El objetivo de estudio fue analizar el lexema neur(o) y los términos anatómicos asociados con el fin de comprender su significado y mejorar su aprendizaje. Para ello, se identificaron los términos anatómicos que contienen en su composición el lexema neur(o) en Terminologia Anatomica (TA) publicada el 2011 y analizados etimológica y semánticamente. El lexema neur $(o)$ se repite en 26 términos anatómicos provenientes del latín y griego en los capítulos sistema esquelético (A02.0.00.000 Ossa; Systema skeletale), sistema muscular (A04.0.00.000 Musculi, Systema musculare), glándulas endocrinas (A11.0.00.000 Glandulae endocrinae) y sistema nervioso (A14.0.00.000 Systema nervosum). La raíz neur(o), tiene dos orígenes semánticos: del griego veṽ pov, como también del latín neruu $(m)$, cuyos significados son nervio o tendón, como en el caso de "aponeurosis" (A04.0.00.47 Aponeurosis),

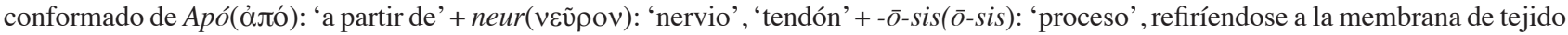
conjuntivo que envuelve los músculos. En conclusión, los términos anatómicos en TA poseen un origen grecolatino que hace interesante el aprendizaje, proporcionando adherencia en el proceso de aprendizaje de cada término anatómico.
\end{abstract}

Palabras Clave: Neuro, neuroanatomía; Terminologia Anatomica; Aponeurosis.

\section{INTRODUCCIÓN}

Una de las fronteras hacia donde avanza el conocimiento humano, inmersa en lo complejo y carente de teorías generales, es en un fenómeno singular llamado mente (García Valdecasas, 1980). Durante los siglos XVII y XVIII se plantea la unión entre mente y sistema nervioso (Duque-Parra, 2002). En este contexto, la neuroanatomía y la neurofisiología, hasta la primera mitad del siglo $\mathrm{XX}$, fueron las ramas de las ciencias básicas que más aportaron al conocimiento fundamental de este sistema. El desarrollo técnico, bioquímico, informático e imaginológico, entre otros, favorecieron una unión morfofisiológica que permitió, a partir de los años 60, dar solidez a los conocimientos en esta área (Duque-Parra, 2001).
Estos avances, sumados a la importancia que fue adquiriendo la neuroanatomía, la convirtieron en una de las ramas más complejas de abordar por parte de los estudiantes. Quienes deben familiarizarse con un lenguaje complejo y extenso, necesario para lograr una comunicación global (Aziz et al., 2002). Así, la comprensión del término neuro desde su origen podría favorecer el aprendizaje de esta materia. Neuro proviene del indoeuropeo nerwo, que deriva de neu-ro (de neu -variante de sneu-), que significa liga. Tardíamente, la familiaridad del concepto es clara, "nervio", procedente del latín vulgar nervus, del griego veũpov, nervio, tendón (Hernández-Alcántara, 2011).

\footnotetext{
${ }^{1}$ Escuela de Enfermería, Facultad de Salud, Universidad Santo Tomás, Chile.

${ }^{2}$ Becario CONICYT-PCHA/MAGISTER NACIONAL/ 2017-22170070.

${ }^{3}$ Programa de Magíster en Ciencias mención Morfología, Universidad de La Frontera, Temuco, Chile.

${ }^{4}$ Programa de Magíster en Lingüística Aplicada, Facultad de Humanidades y Arte, Universidad de Concepción, Concepción, Chile.

${ }^{5}$ Departamento de Ciencias Morfológicas, Facultad de Medicina y Ciencia, Universidad San Sebastián, General Lagos 1163, Valdivia 509000, Chile.

${ }^{6}$ Programa de Doctorado en Ciencias Morfológicas, Universidad de La Frontera, Temuco, Chile.

${ }^{7}$ Departamento de Ciencias de la Salud, Universidad de Aysén, Coyhaique, Chile.

${ }^{8}$ Núcleo de Idiomas, Facultad de Estudios Interdisciplinarios, Universidad Mayor, Temuco, Chile.
} 
La importancia de comprender el lexema neur(o) y los términos que derivan de él, radica en la multiplicidad de conceptos que lo tiene incorporado como prefijo, sufijo o interfijo. Por ende, determinar las unidades mínimas de cada palabra, definidas como raíz o lexema, permitirá descifrar el sentido semántico de una palabra y los morfemas que derivan de ella. Desde un punto de vista lexicogenésico, las palabras se construyen adicionando alternativas de composición pudiendo ser de tres tipos: prefijos, sufijos e interfijos. Los prefijos son los que se anteponen a la base lexical, los sufijos se posponen al lexema y los interfijos los que se intercalan entre la raíz y los sufijos (Marcos, 1998).

Por otra parte, Terminologia Anatomica (TA) es la encargada de unificar todos los términos anatómicos empleados para nombrar, identificar, describir y ubicar espacialmente a los órganos corporales, así como las relaciones entre ellos. (Aziz et al.; Díaz Rojo, 2001). En 1989, la Federación Internacional de Asociaciones de Anatomía (IFAA), estableció que TA sea redactada en latín y usada como base de la comunidad científica internacional. Los términos anatómicos allí incluidos poseen sus orígenes en la antigua Grecia y Roma (Sakai, 2007), con lo que cuentan con una fuerte influencia del latín clásico y el griego (Wulff, 2004). Considerando la información expuesta, el objetivo de esta investigación fue analizar el lexema neur(o) y los términos anatómicos asociados con el propósito comprender su significado y mejorar su aprendizaje.

\section{MATERIAL Y MÉTODO}

Se identificaron los términos anatómicos que contienen en su composición el lexema neur(o) en TA (2011) publicada por el Programa Federativo Internacional en Terminología Anatómica (FIPAT), ubicados en la columna en latín. Cada uno de estos términos fue analizado desde un punto de vista etimológico y semántico según las directrices propuestas por el Diccionario Médico-Biológico, Histórico y Etimológico de la Universidad de Salamanca (2014). Finalmente, con todos los datos recolectados, se construyó una Tabla.

\section{RESULTADOS}

La revisión de la segunda edición de la TA de 2011 en latín e inglés, arrojó que el lexema neur $(o)$ se repite en 26 términos anatómicos (Tabla I). Estos se encuentran en los capítulos sistema esquelético (A02.0.00.000 Ossa; Systema skeletale), sistema muscular (A04.0.00.000 Musculi,
Systema musculare), glándulas endocrinas (A11.0.00.000 Glandulae endocrinae) y sistema nervioso (A14.0.00.000 Systema nervosum).

La raíz neur(o), proviene del griego veṽpov. Qué significa nervio o tendón. En este último, su traducción se hace pertinente en el concepto "aponeurosis" (A04.0.00.47 Aponeurosis), término griego conformado por ảjó 'a partir

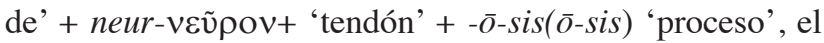
cual hace referencia a la membrana de tejido conjuntivo que envuelve los músculos (Cortés) o como tendones que se extienden en forma de amplias láminas fibrosas (Pró, 2012). En dos casos asociados a glándulas endocrinas, neurohipófisis (A11.1.00.006 Neurohypophysis; Lobus posterior) y lóbulo nervioso (A11.1.00.008 Lobus nervosus), en donde el prefijo neur $(o)$ hace referencia al origen de las estructuras.

\section{DISCUSIÓN}

Si bien, la interiorización de los términos anatómi$\cos$, en principio, puede provocar cierta resistencia debido a que el lenguaje de esta disciplina es bastante amplio. Al comprender los términos desde sus orígenes, estos adquieren sentido y mayor comprensión (Moore et al., 2010).

Uno de los principales resultados de esta investigación fue la diferencia entre la definición otorgada por TA y el origen lexicológico, para los términos aponeurosis y neurofibrarum (Tabla I), quienes poseen una utilización anatómica muy distintas, pero comparten un mismo origen etimológico. Al respecto, es importante desarrollar aspectos históricos que podrían tener injerencia sobre estos dos términos.

Alcmeón de Crotona (500-450 a.C.), basándose en una disección del sistema nervioso, ya pudo distinguir los nervios sensoriales de los motores (Duque-Parra et al., 2014). En paralelo, Empédocles (495-444 a.C.), determinó que los ligamentos o neura eran cordeles o cuerdas (fibras torcidas) que "ataban" el cerebro con el resto de los órganos corporales (Campohermoso et al., 2016). Años más tarde, Herófilo (335-280 a.C.) confundió, por su semejanza, ligamentos y nervios, aunque también reconoció que estos últimos desempeñaban un papel primordial en los procesos sensoriales (Hernández Alcántara, 2011). Por último, Galeno con sus excelentes conocimientos sobre neuroanatomía, aclaró la diferencia entre nervio, ligamento y tendón. Al respecto decía: "nervio, ligamento y tendón, frecuentemente se confunden entre sí. El nervio, por una parte, es lo que emerge del cerebro o la médula espinal, y, por otra parte, el ligamento nace del hueso y el tendón es de 
Tabla I. Casos de la utilización del lexema griego neur(o) en Terminologia Anatomica, su origen y significado en español.

\begin{tabular}{|c|c|c|c|}
\hline Código & Latín & Origen & Español \\
\hline A02.1.00.007 & Neurocranium & $\begin{array}{l}\text { Griego: neur(o)- veũoov 'nervio', 'sistema nervioso' + } \\
\text { krān(io)- kpqvíov 'cabeza' }\end{array}$ & Neurocraneo \\
\hline A04.0.00.47 & Aponeurosis & 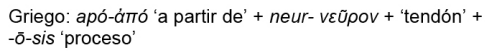 & Aponeurosis \\
\hline A04.1.03.007 & $\begin{array}{l}\text { Galea apeneurotica; } \\
\text { Aponeurosis } \\
\text { epicrenialis }\end{array}$ & 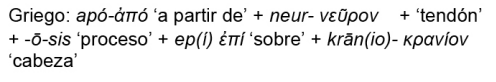 & Aponeurosis epicraneal \\
\hline A04.3.02.003 & $\begin{array}{l}\text { Aponeurosis musculi } \\
\text { erectoris spine }\end{array}$ & 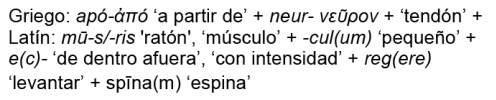 & $\begin{array}{l}\text { Aponeurosis del músculo } \\
\text { erector de la columna }\end{array}$ \\
\hline A04.6.02.016 & $\begin{array}{l}\text { Aponeurosis musculi } \\
\text { bicipitis brachii }\end{array}$ & 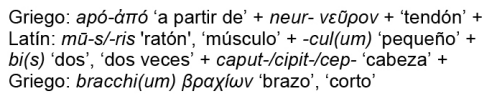 & $\begin{array}{l}\text { Aponeurosis del músculo } \\
\text { bíceps braquial }\end{array}$ \\
\hline A04.6.03.012 & Aponeurosis palmaris & $\begin{array}{l}\text { Griego: apó-d̆mó 'a partir de' + neur- veũpov + 'tendón' + } \\
\text { Latín: palma 'palma' }\end{array}$ & Aponeurosis palmar \\
\hline A04.7.02.009 & Aponeurosis glutea & 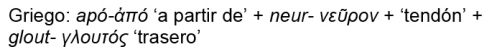 & Aponeurosis glútea \\
\hline A04.7.03.031 & Aponeurosis plantaris & $\begin{array}{l}\text { Griego: apó-ámó 'a partir de' + neur- veũpov + 'tendón' + } \\
\text { Latín: planta(m) 'planta del pie', 'esqueje' }\end{array}$ & Aponeurosis plantar \\
\hline A05.1.04.025 & Aponeurosis linguae & $\begin{array}{l}\text { Griego: apó-ámó 'a partir de' + neur- veũpov + 'tendón' + } \\
\text { Latín: lingua }(m) \text { 'lengua' }\end{array}$ & Aponeurosis lingual \\
\hline A05.2.01.101 & Aponeurosis palatina & $\begin{array}{l}\text { Griego: apó-ámó ‘a partir de' + neur- veũoov + 'tendón' + } \\
\text { Latín: palāt(um) 'paladar' }\end{array}$ & Aponeurosis palatina \\
\hline A11.1.00.006 & $\begin{array}{l}\text { Neurohypophysis; } \\
\text { Lobus posterior }\end{array}$ & 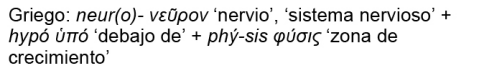 & Neurohipofisis; lóbulo posterior \\
\hline A11.1.00.008 & Lobus nervosus & $\begin{array}{l}\text { Griego: Iob(o)- גoßós 'lóbulo' + Latín: neruu(m) 'nervio', } \\
\text { 'tendón' + -ōs(um)/-ōs(am) 'abundancia' }\end{array}$ & Lóbulo nervioso \\
\hline A14.0.00.001 & Neurofibra & $\begin{array}{l}\text { Griego: neur(o)- vẽ̃oov 'nervio', 'sistema nervioso' + } \\
\text { Latín: fibr(am) 'fibra' }\end{array}$ & Fibra nerviosa \\
\hline A14.0.00.002 & Neuron & 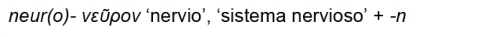 & Neurona \\
\hline A14.0.00.005 & Neuroglia & $\begin{array}{l}\text { Griego: neur(o)- veũpov 'nervio' + 'sistema nervioso' + } \\
\text { gli(a) VAfia 'pegamento' }\end{array}$ & Neuroglia \\
\hline A14.1.09.320 & $\begin{array}{l}\text { Neurofibrae } \\
\text { tangentiales }\end{array}$ & $\begin{array}{l}\text { Griego: neur(o)- veũpov 'nervio', 'sistema nervioso' + } \\
\text { Latín: fibr(am) 'fibra' + tangentis 'el que toca' + ia + al } \\
\text { 'relativo a'. }\end{array}$ & Neurofibra tangencial \\
\hline A14.2.00.009 & $\begin{array}{l}\text { Neurofibrae } \\
\text { preganglionicae }\end{array}$ & 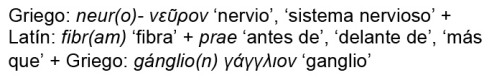 & $\begin{array}{l}\text { Fibras nerviosas } \\
\text { preganglionares }\end{array}$ \\
\hline A14.2.00.010 & $\begin{array}{l}\text { Neurofibrae } \\
\text { postganglionicae }\end{array}$ & $\begin{array}{l}\text { Griego: neur(o)- veũpov 'nervio', 'sistema nervioso' + } \\
\text { Latín: fibr(am) 'fibra' + post 'después de', 'detrás de' + } \\
\text { Griego: gánglio(n) yớyyגıov 'ganglio' }\end{array}$ & $\begin{array}{l}\text { Fibras nerviosas } \\
\text { postganglionares }\end{array}$ \\
\hline A14.2.00.014 & Endoneurium & 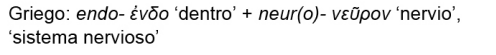 & Endoneuro \\
\hline A14.2.00.015 & Perineurium & 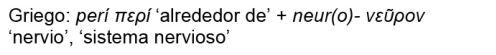 & Perineuro \\
\hline A14.2.00.016 & Epineurium & 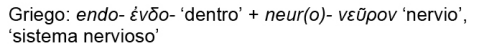 & Epineuro \\
\hline A14.2.00.017 & $\begin{array}{l}\text { Neurofibrae } \\
\text { afferentes }\end{array}$ & $\begin{array}{l}\text { Griego: neur(o)- vẽopov 'nervio', 'sistema nervioso' + } \\
\text { Latín: fibr(am) 'fibra' + ad 'junto a', 'hacia' + fer(re) 'llevar' } \\
+- \text {-nte }(m) \text { 'que hace' }\end{array}$ & Fibras nerviosas aferentes \\
\hline A14.2.00.018 & $\begin{array}{l}\text { Neurofibrae } \\
\text { efferentes }\end{array}$ & $\begin{array}{l}\text { Griego: neur(o)- vẼopov 'nervio', 'sistema nervioso' + } \\
\text { Latín: fibr(am) 'fibra' + e(c)- 'de dentro afuera', 'con } \\
\text { intensidad' + fer(re) 'llevar' + -nte(m) 'que hace' }\end{array}$ & Fibras nerviosas eferentes \\
\hline A14.2.00.019 & $\begin{array}{l}\text { Neurofibrae } \\
\text { somaticae }\end{array}$ & 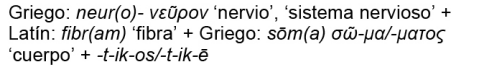 & Fibras nerviosas somáticas \\
\hline A14.2.00.020 & $\begin{array}{l}\text { Neurofibrae } \\
\text { autonomicae }\end{array}$ & 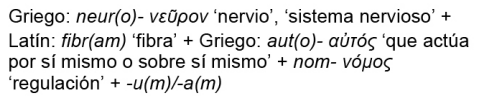 & Fibras nerviosas autónomas \\
\hline A15.2.04.017 & $\begin{array}{l}\text { Stratum } \\
\text { neurofibrarum }\end{array}$ & $\begin{array}{l}\text { Latin: stratus 'estrato' + Griego: neur(o)- veũpov 'nervio', } \\
\text { 'sistema nervioso' + Latín: fibr(am) 'fibra' }\end{array}$ & Capa de las fibras nerviosas \\
\hline
\end{tabular}

donde se origina un músculo" (Campohermoso et al., 2016).

En la actualidad, nervio se define como "cordón de haces de fibras nerviosas, integrante fundamental del sistema nervioso periférico, que conduce impulsos nerviosos hacia o desde el sistema nervioso central o en ambos sentidos" (Cortés, 2014). De manera simplificada, podemos decir que este tejido tiene forma de cordón, es conductor de impulsos y está compuesto de fibras. Por tanto, esta condición de "cordón de haces de fibras" podría haber generado una confusión entre nervio y tendón en un pasado.

Aclarado el origen del término neur(o) y retomando el análisis etimológico de la palabra aponeurosis se puede apreciar cierta inconsistencia entre su origen y su utilización. Para esto es necesario contrastar tres puntos; i) Apó(àró): 'a

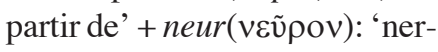
vio', 'tendón' + - $\bar{o}$-sis( $\bar{o}$-sis $)$ : 'proceso', ii) En la terminología anatómica es utilizado para referirse a una membrana de tejido conjuntivo que envuelve los músculos y iii) Galeno (II d.C.), referido al punto en el que el músculo se hace tendinoso (Diccionario de términos médicos, 2011). En este contexto, el sufijo -ō-sis( $\bar{o}$-sis): 'proceso' generaría confusión, entendiendo a la aponeurosis como un conjunto de membranas fibrosas que envuelven a los músculos característica distinta a un proceso óseo (Testut \& Latarjet, 1983).

Por otra parte, el resto de los resultados mostró consistencia entre el origen de la palabra y su aplicación. A modo de ejemplo, el término en latín 
neurocranium está formado por dos lexemas. El lexema griego neur(o) (nervio, sistema nervioso) y lexema en latín medieval crāneu(m) (cabeza). Desde su significado, Cortés (2014) lo define como una caja ósea donde está contenido el encéfalo. Esto concuerda con su aplicación anatómica, aquí Alves \& Cândido (2009) describen esta estructura como la porción del cráneo compuesta por ocho huesos que envuelven y protegen la parte cefálica del sistema nervioso central, el encéfalo. Por tanto, existe una directa relación entre origen, significado y aplicación.

Del mismo modo, el término neurohypophysis es empleado para referirse a una zona específica de la hipófisis, glándula ubicada bajo el cerebro. La neurohipofisis corresponde a una extensión del tejido nervioso del cerebro, la cual secreta neurohormonas producidas en el hipotálamo (Silverthorn \& Ober, 2010). Esta caracterización concuerda con lo expuesto por el Diccionario de términos médicos, (2011), donde se define como la porción nerviosa de la hipófisis, que deriva del suelo del tercer ventrículo. Contiene las terminaciones axónicas del tracto supraóptico hipofisiario, que liberan las hormonas vasopresina y oxitocina sintetizadas por las neuronas de gran tamaño de los núcleos supraóptico y paraventricular del hipotálamo. Del mismo modo, los términos neurona (neuron) y neuroglia (neuroglia) (Tabla I) son utilizados para referirse a células constitutivas del sistema nervioso. Muchos especialistas en el área, utilizan el término neurona para referirse a la unidad funcional del sistema nervioso, la cual tiene propiedades de excitabilidad y conductibilidad (Duque-Parra, 2001; Standring, 2016). Asimismo, el grupo de células que sostienen a esta unidad funcional, se denomina neuroglia (Snell, 2010; Moore et al.).

Los términos neurofibra, neurofibrae, neurofibrarum han sido traducidos por $T A$ al español, en el caso del primero, como fibra nerviosa y el segundo como su plural, fibras nerviosas. Situación especial es la de neurofibrarum que es el genitivo de neurofibra por lo que su definición sería, de las fibras nerviosas. Esta definición se asemeja a lo descrito por Ross \& Pawlina, (2016), quienes utilizan el término "fibra nerviosa" para referirse al axón con todas sus cubiertas, es decir, cada filamento que entra en la composición de un axón. Los términos endoneurium, perineurium y epineurium son empleados para describir el tejido conectivo que cubre a los nervios periféricos, endoneuro, perineuro y epineuro. El uso de los prefijos endo (dentro), peri (alrededor de) y epi (sobre), permiten atribuir una ubicación de estos tejidos en el nervio. El epineuro corresponde a la capa más externa del nervio, el perineuro corresponde a una capa de tejido conectivo especializado que se localiza alrededor de cada fascículo nervioso y el endoneuro corresponde a una capa de tejido conectivo laxo localizado alrededor de cada fibra nerviosa (Ross \& Pawlina; Moore et al.)

\section{CONCLUSIÓN}

Los principales hallazgos de esta investigación fueron que el sufijo -ō-sis generó confusión en el significado del término aponeurosis. Los términos anatómicos en $T A$ poseen un origen grecolatino que hace muy interesante el aprendizaje, pues lo plantea desde el sentido primigenio del concepto, con lo cual, se enriquece el conocimiento de la palabra, proporcionando adherencia en el proceso de acercamiento y aprehensión del lenguaje, que en este caso es el término anatómico.

En este estudio, se demostró que el conocimiento de un solo lexema permite inferir la definición y aplicación de 26 términos anatómicos, por lo que se ratifica la importancia de la comprensión básica de las etimologías latinas y griegas en la enseñanza y aprendizaje de la anatomía. El tratamiento de un lexema y su recurrencia, refuerzan y demuestran la necesidad de que la formación en salud, deben incorporar contenidos curriculares de formación lexicogenesia grecolatina, para facilitar la aproximación a las disciplinas que la componen, fortaleciendo y unificando el lenguaje científico de la comunidad nacional e internacional.

VIDAL-SEGUEL, N.; PUMEYRAU, S. M.; LIZAMA, P. R.; MUÑOZ, C. R.; CONEI, D.; NICHOLSON, C.\& DEL SOL, M. Analysis of neur(o) lexeme and its derivatives in anatomy learning. Int. J. Morphol., 37(4):1517-1521, 2019.

SUMMARY: Neuroanatomy is one of the most complex areas addressed by students because of its complex and extensive language. That is why the term neuro could favor its learning, because multiple concepts and terms have it incorporated as a prefix, suffix or interfix. The aim of study was to analyze neur $(o)$ lexeme and the associated anatomical terms in order to understand its meaning and improve its learning. For this, the anatomical terms that contain neur (o) lexema in Terminologia Anatomica (TA) published in 2011 and analyzed etymologically and semantically were identified in its composition. The neur (o) lexeme is repeated in 26 anatomical terms from latin and greek in the skeletal system (A02.0.00.000 Ossa; Systema skeletale), muscular system (A04.0.00.000 Musculi, Systema musculare), endocrine glands (A11.0.00.000 Glandulae endocrinae) and nervous system (A14.0.00.000 Systema nervosum) chapters. The neur $(o)$ root has two semantic origins: from greek veupov, as well as from latin $\operatorname{neruu}(\mathrm{m})$, whose meanings are nerve or tendon, as in the case of "aponeurosis" (A04.0.00.47 Aponeurosis), formed by

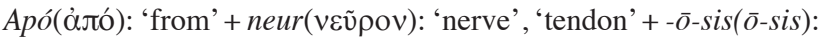
'process', referring to the connective tissue membrane that surrounds the muscles. In conclusion, the anatomical terms in $T A$ have a greek and latin origin that makes learning interesting, providing adherence in the learning process of each anatomical term.

KEY WORDS: Neuro; Neuroanatomy; Terminologia Anatomica; Aponeurosis. 


\section{REFERENCIAS BIBLIOGRÁFICAS}

Alves, N. \& Cândido, P. Anatomía aplicada a la odontología. Sao Paulo: Livraria Santos, 2009.

Aziz, M. A.; McKenzie, J. C.; Wilson, J. S.; Cowie, R. J.; Ayeni, S. A. \& Dunn, B. K. The human cadaver in the age of biomedical informatics. Anat. Rec., 269(1):20-32, 2002.

Campohermoso Rodríguez, O. F.; Soliz Soliz, R. E.; Campohermoso Rodríguez, O. \& Zúñiga Cuno, W. Galeno de Pérgamo "Príncipe de los médicos”. Cuad. Hosp. Clín., 57(2): 84-93, 2016.

Comité Federal sobre Terminología Anatómica (FCAT). Terminologia Anatomica. Terminología Anatómica Internacional. Madrid, Panamericana, 2001.

Cortés, G.F. Diccionario Médico-Biológico, Histórico y Etimológico. Salamanca. Universidad de Salamanca, 2014. Disponible en: http:// www.dicciomed.es

Díaz Rojo, J.A. La terminología médica: diversidad, norma y uso. Med. Interam., 2(4):40-6, 2001.

Duque-Parra, J.E. Functional neuroanatomy: the first daughter of neuroscience and the mother of neural science. Anat. Rec., 265(6):250-3, 2001.

Duque-Parra, J.E. Elementos neuroanatómicos y neurológicos asociados con el cerebro a través del tiempo. Rev. Neurol., 34(3):282-286, 2002.

Federative International Committee on Anatomical Terminologies (FICAT). Terminologia Anatomica. International Anatomical Terminology. New York, Thieme, 1998.

Federative International Programme on Anatomical Terminologies (FIPAT). Terminologia Anatomica. $2^{\text {a }}$ ed. New York, Thieme, 2011.

García Valdecasas, J. Evolución de los conceptos sobre el funcionamiento cerebral. Facultad de Medicina. Universidad Nacional Autónoma de México, 1980.

Hernández Alcántara, A. Acerca de la etimología de "nervosa" en la bulimia y anorexia: una historia de nervios. Enseñanza e Investigación en Psicología,16(2):387-394, 2011.

Marcos MarÍn, F. Gramática española. $2^{\mathrm{a}}$ ed España: Editorial Síntesis, 1998.

Moore, K.L.; Dalley, A.F.; Agur, A.M.R. Anatomía con orientación Clínica. 6 Ed. España: Lippincott Williams \& Wilkins, 2010.

Pró, E.A. Anatomía Clínica. 2 Ed. Buenos Aires, ed. Médica Panamericana, 2012.

Real Academia Nacional de Medicina. Diccionario de Términos Médicos. Buenos Aires. Ed. Médica Panamericana, 2011.

Ross, M. \& Pawlina, W. Histología: Texto y atlas. Correlación con Biología celular y molecular. $7^{\mathrm{a}}$ ed. España: Wolver Kluger, 2016.

Sakai, T. Historical evolution of anatomical terminology from ancient to modern. Anat. Sci. Int., 82(2):65-81, 2007.

Silverthorn, D. \& Ober, W. Fisiología humana. $4^{\mathrm{a}}$ ed. Buenos Aires. Ed. Médica Panamericana, 2010.

Smith, S.B.; Carmichael, S.W.; Pawlina, W. \& Spinner, R.J. Latin and Greek in gross anatomy. Clin. Anat., 20(3):332-7, 2007.

Snell, R. Neuroanatomía Clínica. $7^{\mathrm{a}}$ ed. Barcelona: Lippincott Williams \& Wilkins, 2010

Standring, S. Gray's Anatomy: The Anatomical Basis of Clinical Practice. $41^{\text {a }}$ ed. New York, Elsevier Limited, 2016.

Testut, L. \& Latarjet, A. Anatomía Humana. Barcelona, Salvet Editores, 1983.

Wulff, H.R. The language of medicine. J.R. Soc.Med., 97(4):187-8, 2004.

\author{
Dirección para correspondencia: \\ Nicolás Enrique Vidal Seguel \\ Universidad Santo Tomás \\ Mendoza 120 \\ Los Ángeles \\ CHILE
}

Email: nvidal4@santotomas.cl

Recibido : 15-04-2019

Aceptado: 23-06-2019 